\title{
Psychologische Psychotherapie: Datenlücke geschlossen
}

Bis vor kurzem gab es nur bruchstückhafte Informationen zu Angebot, Inanspruchnahme, Kosten und Finanzierung der psychologischen Psychotherapie in der Schweiz. Eine im Auftrag der Föderation der Schweizer Psychologinnen und Psychologen (FSP) durchgeführte Studie liefert nun Daten und belegt, dass psychologische Psychotherapeut(inn)en eine wichtige Rolle in der Behandlung psychischer Erkrankungen spielen und dazu beitragen können, der Versorgungslücke entgegenzuwirken.

\section{Yvik Adler ${ }^{a}$, Philipp Thüler ${ }^{b}$}

a lic. phil., Fachpsychologin für Psychotherapie FSP, Vizepräsidentin der Föderation der Schweizer Psychologinnen und Psychologen FSP

b Stellvertretender Leiter des Bereichs Kommunikation und Marketing der FSP

Interessenverbindungen: Die Studie wurde hauptsächlich durch die FSP finanziert.

Lesen Sie hierzu auch den nachfolgenden Kommentar der FMPP.
Psychologische Psychotherapeutinnen und -therapeuten sind unabdingbare Leistungserbringer(innen) im schweizerischen Gesundheitssystem. In Anbetracht des Nachwuchsmangels bei den Psychiaterinnen und Psychiatern, der in dieser Zeitschrift auch schon thematisiert wurde [1], scheint es klar, dass ohne adäquaten Einbezug der psychologischen Psychotherapie ein Versorgungsengpass droht.

Seit April 2013 ist das eidgenössische Psychologieberufegesetz (PsyG) in Kraft, in dem die Bedingungen zur Ausübung der psychologischen Psychotherapie klar geregelt sind. Das PsyG schützt den Titel des psychologischen Psychotherapeuten/der psychologischen Psychotherapeutin. Eine logische Konsequenz muss die Ablösung des Modells der delegierten Psychotherapie sein. Das Bundesamt für Gesundheit (BAG) prüft deshalb zurzeit neue Modelle der Zulassung der psychologischen Psychotherapie zur Grundversicherung.

\section{Anordnung statt Delegation?}

Das bestehende Modell der delegierten Psychotherapie soll von dem zurzeit diskutierten Anordnungsmodell abgelöst werden. Gemäss dem Anordnungsmodell würde der/die psychologische Psychotherapeut(in) psychotherapeutische Behandlungen, die von einem Arzt/einer Ärztin verordnet sind, in eigener Verantwortung durchführen und selbständig über die Grundversicherung abrechnen.

\section{Solide Datengrundlage}

Um die Diskussionen rund um ein neues Versorgungsmodell auf einer soliden und aktuellen Datengrundlage führen zu können, hat die FSP das unabhängige Büro für arbeits- und sozialpolitische Studien BASS mit der Durchführung einer breitangelegten Studie beauftragt. Die Erhebung wurde von den weiteren Berufsverbänden SBAP (Schweizerischer Berufsverband für Angewandte Psychologie) und ASP (Assoziation Schweizer PsychotherapeutInnen und Psychotherapeuten) und deren Mitgliedern sowie von der SGPP (Schweizerischen Gesellschaft für Psychiatrie und Psy- chotherapie), der FMPP (Dachorganisation der psychiatrisch-psychotherapeutisch tätigen ÄrztInnen der Schweiz) und der SVPC (Schweizerischen Vereinigung Psychiatrischer Chefärztinnen und Chefärzte) unterstützt.

Der nun vorliegende Schlussbericht* liefert Daten dazu, wie viele psychologische Psychotherapeutinnen und -therapeuten in der Schweiz in ihrem Beruf tätig sind, wie und von wem ihre Leistungen in Anspruch genommen werden, was diese Leistungen kosten, und wie sie finanziert werden.

\section{Personen befragt}

Die Erhebung erfolgte in zwei Schritten: Zunächst wurde eine Grundgesamtheit von rund 9100 Psychologinnen und Psychologen befragt. Ziel dieser Vollerhebung war es zu erfahren, wie viele Psychologinnen und Psychologen psychotherapeutisch tätig sind, und wenn ja, in welcher Arbeitsform (selbständig, delegiert, in einer ambulanten oder stationären Institution) und in welchem Umfang.

In einem zweiten Schritt wurde aus der Gruppe von rund 4000 psychotherapeutisch tätigen Psychologinnen und Psychologen eine Stichprobe von knapp 2200 Personen zu den im Jahr 2012 erbrachten psychotherapeutischen Leistungen befragt. Dank hoher Rücklaufquoten von $67 \%$ bei der Vollerhebung und 53\% bei der Stichprobenerhebung kann von einer sehr guten Datenqualität ausgegangen werden. Anhand von zusätzlichen Daten, die freundlicherweise von der SASIS AG, einer Tochterfirma der santésuisse, zur Verfügung gestellt wurden, konnten die Ergebnisse mit den Kosten der Grundversicherung verglichen und plausibilisiert werden. Der Vergleich zeigt eine sehr gute Übereinstimmung, was für eine hohe Datenvalidität spricht. Zusätzlich ergänzt wurden die Erhebungsdaten mit den Daten zur Zusatzversicherung eines grossen Krankenversicherers.

\section{0 psychologische Psychotherapeut(inn)en} Gemäss dem Schlussbericht erbringen in der Schweiz rund 5700 psychologische Psychotherapeutinnen und 
Abbildung 1

Altersstruktur nach Arbeitsform.

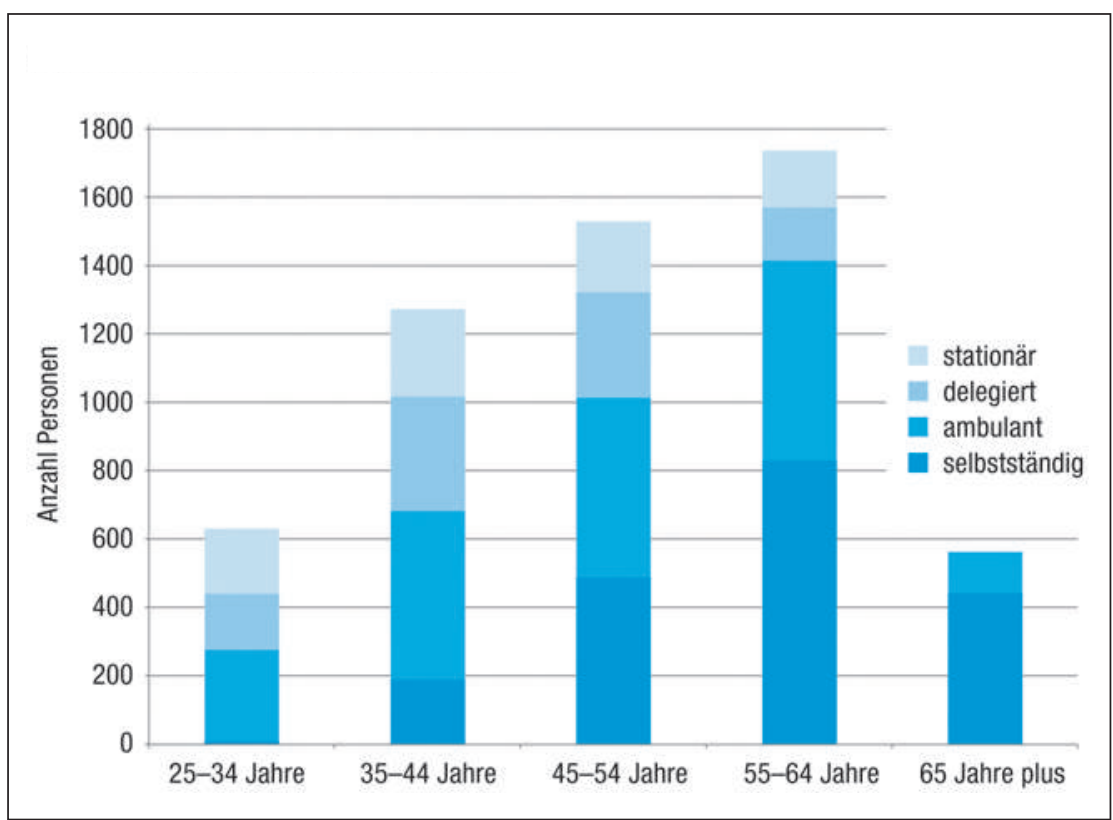

-therapeuten psychotherapeutische Leistungen im Umfang von etwa 3100 Vollzeitstellen. Die durchschnittlichen Arbeitspensen betragen 70 bis 90 Prozent, wobei in dieser Zahl auch der Aufwand für Administration, Führungsaufgaben, eigene Supervision und Weiterbildung enthalten ist.

34\% des Angebots wurde in selbständiger Praxis, $31 \%$ in delegierter Praxis und 36\% in Institutionen erbracht. Erwartungsgemäss arbeiteten jüngere Psychotherapeutinnen und -therapeuten eher delegiert oder in einer Institution, während der grösste Teil der in selbständiger Praxis tätigen Psychotherapeutinnen und -therapeuten in der Altersgruppe der 55-64-Jährigen lag (Abb. 1). Der Anteil der Psychotherapeutinnen und -therapeuten mit Sprachkenntnissen auf muttersprachlichem Niveau war je nach Region mit 90 bis 98\% bemerkenswert hoch.

Bezüglich Geschlechterverteilung ist zu erwähnen, dass 75\% der psychotherapeutisch Tätigen weiblich waren, 25\% männlich. Das Spektrum an Psychotherapiemethoden war dabei breit und vielfältig. 55\% der psychologischen Psychotherapeut(inn)en hatten zusätzlich eine zweite oder mehrere psychotherapeutische Weiterbildungen absolviert. In der Studie wird der Nachweis erbracht, dass psychologische Psychotherapeut(inn)en bereits vor Inkraftreten des Psychologieberufegesetzes auf hohem Niveau für die psychotherapeutische Behandlung von psychisch kranken Menschen ausgebildet waren

\section{Im Schnitt 29 Konsultationen}

Die psychologischen Psychotherapeutinnen und -therapeuten erbrachten 2012 in der Schweiz rund 3,1 Millionen Konsultationen für 260000 Patientinnen und Patienten.
Rund 60 Prozent der Patientinnen und Patienten wurden in einem Setting von zwei bis zwanzig Sitzungen im Jahr behandelt. 21 Prozent der Patientinnen und Patienten begaben sich für 21 bis 40 Sitzungen in Behandlung. Nur ein sehr kleiner Teil von vier Prozent der Patientinnen und Patienten benötigte mehr als 40 Konsultationen pro Jahr (Tab. 1). Die durchschnittliche Therapiedauer betrug 29 Konsultationen, dies über einen durchschnittlichen Zeitraum von 15 Monaten.

Das Diagnosespektrum ist aus Tabelle 2 zu entnehmen. Bei den Diagnosen der behandelten Patienten fand man 25\% affektive Störungen, 25\% Anpassungsstörungen, $15 \%$ Störungen im Kindes-und Jugendalter, 11\% Persönlichkeitsstörungen, 7\% Abhängigkeitserkrankungen und 3\% Erkrankungen aus dem schizophrenen Formenkreis. 41\% aller Patienten, die von psychologischen PsychotherapeutInnen behandelt wurden, wiesen komorbide psychische Störungen auf.

Zusammengefasst kann man sagen, dass der Nachweis erbracht wurde, dass die psychologischen Psychotherapeuten ein vielfältiges Therapieangebot für den gesamten Bereich psychischer Störungen bereitstellen und auch Menschen mit schweren psychischen Erkrankungen behandeln.

\section{Zugang zur Psychotherapie}

Etwa die Hälfte der psychologischen Psychotherapeutinnen und -therapeuten gab an, dass im Jahr 2012 Wartefristen für neue Patientinnen und Patienten bestanden und/oder sie Patientinnen und Patienten aus Kapazitätsgründen abweisen mussten. 16 Prozent der Patientinnen und Patienten konnten die Therapie aus Kostengründen nicht antreten oder mussten sich einen über die Grundversicherung finanzierten Therapieplatz suchen, und 22 Prozent der Therapien mussten aus finanziellen Gründen abgebrochen werden. Nicht erfasst sind Personen, die sich im Wissen um die Kostenfolgen gar nicht erst um eine psychologische Psychotherapie bemüht hatten.

\section{Hohe Selbstzahlerquote}

Die Kosten für Leistungen durch psychologische Psychotherapeutinnen und -therapeuten im ambulanten Bereich beliefen sich 2012 auf 406 Millionen Franken. Der grösste Teil dieser Kosten wurde mit rund 270 Millionen Franken von den Sozialversicherungen getragen, wovon die Grundversicherung mit rund 246 Millionen den Hauptteil übernahm. 23 Millionen Franken wurden über die Invalidenversicherung abgerechnet. 117 Millionen Franken respektive knapp 30 Prozent der Gesamtkosten der psychologischen Psychotherapie im ambulanten Bereich wurden privat getragen oder über Zusatzversicherungen abgerechnet. Hochrechnungen zeigen, dass 2012 mit rund 18 Millionen Franken nur ein sehr kleiner Teil der Kosten der psychologischen Psychotherapie über Zusatzversicherungen abgerechnet wurde. Ein grösserer Anteil der Kosten - rund 100 Millionen Franken - wurde hingegen von den Patientinnen und Patienten selber getragen. 
Tabelle 1

Anteil der Patient(inn)en nach Anzahl Therapiesitzungen im Jahr 2012, je Arbeitsform.

\begin{tabular}{lccccc} 
& in einer Praxis & \multicolumn{2}{c}{ in einer Institution } & Gesamt \\
\hline & selbständige PT & delegierte PT & ambulant & stationär & \\
\hline 1 Sitzung / Erstgespräch & $12 \%$ & $9 \%$ & $21 \%$ & $27 \%$ & $16 \%$ \\
\hline 2-20 Sitzungen & $59 \%$ & $57 \%$ & $60 \%$ & $59 \%$ & $59 \%$ \\
\hline $21-30$ Sitzungen & $17 \%$ & $18 \%$ & $11 \%$ & $9 \%$ & $14 \%$ \\
\hline $31-40$ Sitzungen & $8 \%$ & $11 \%$ & $5 \%$ & $3 \%$ & $7 \%$ \\
\hline $41-50$ Sitzungen & $3 \%$ & $3 \%$ & $2 \%$ & $2 \%$ & $3 \%$ \\
\hline mehr als 51 Sitzungen & $2 \%$ & $1 \%$ & $1 \%$ & $1 \%$ & $1 \%$ \\
\hline Total & $100 \%$ & $100 \%$ & $100 \%$ & $100 \%$ & $100 \%$ \\
(n= Anzahl antwortende Personen) & $\mathrm{n}=441$ & $\mathrm{n}=373$ & $\mathrm{n}=172$ & $\mathrm{n}=118$ & $\mathrm{n}=1104$
\end{tabular}

Anmerkung: Die Angaben der Tabelle beziehen sich nur auf die 2012 durchgeführten Therapiesitzungen. Es lassen sich keine Rückschlüsse auf die Anzahl Sitzungen je abgeschlossener Psychotherapie ziehen.

(Quelle: Strukturerhebung, Hochrechnung BASS)

Tabelle 2

Anteil der Patient(inn)en je Krankheitsbild gemäss ICD an allen PatientInnen mit Krankheitswert im Jahr 2012, nach Arbeitsform.

\begin{tabular}{|c|c|c|c|c|c|}
\hline \multirow[b]{3}{*}{$\begin{array}{l}\text { Organische, einschliesslich symptomatischer } \\
\text { psychischer Störungen (FO0-FO9) }\end{array}$} & \multicolumn{2}{|c|}{ in einer Praxis } & \multicolumn{2}{|c|}{ in einer Institution } & \multirow[t]{2}{*}{ Gesamt } \\
\hline & selbstän & delegiert & ambulant & stationär & \\
\hline & $2 \%$ & $1 \%$ & $3 \%$ & $2 \%$ & $2 \%$ \\
\hline $\begin{array}{l}\text { Psychische und Verhaltensstörungen durch } \\
\text { psychotrope Substanzen (F10-F19) }\end{array}$ & $2 \%$ & $4 \%$ & $11 \%$ & $13 \%$ & $7 \%$ \\
\hline $\begin{array}{l}\text { Schizophrenie, schizotype und wahnhafte } \\
\text { Störungen (F20-F29) }\end{array}$ & $1 \%$ & $3 \%$ & $3 \%$ & $6 \%$ & $3 \%$ \\
\hline Affektive Störungen (F30-F39) & $23 \%$ & $31 \%$ & $19 \%$ & $33 \%$ & $26 \%$ \\
\hline $\begin{array}{l}\text { Neurotische, Belastungs- und somatoforme } \\
\text { Störungen (F40-F48) }\end{array}$ & $31 \%$ & $30 \%$ & $24 \%$ & $22 \%$ & $27 \%$ \\
\hline $\begin{array}{l}\text { Verhaltensauffälligkeiten mit körperlichen } \\
\text { Störungen und Faktoren (F50-F59) }\end{array}$ & $4 \%$ & $3 \%$ & $3 \%$ & $4 \%$ & $3 \%$ \\
\hline Persönlichkeits- und Verhaltensstörungen (F60-F69) & $10 \%$ & $14 \%$ & $11 \%$ & $8 \%$ & $11 \%$ \\
\hline Intelligenzstörung (F70-F79) & $2 \%$ & $1 \%$ & $2 \%$ & $1 \%$ & $2 \%$ \\
\hline Entwicklungsstörungen (F80-F89) & $3 \%$ & $2 \%$ & $4 \%$ & $1 \%$ & $3 \%$ \\
\hline $\begin{array}{l}\text { Verhaltens- und emotionale Störungen mit Beginn } \\
\text { in der Kindheit und Jugend (F90-F98) }\end{array}$ & $14 \%$ & $10 \%$ & $17 \%$ & $5 \%$ & $12 \%$ \\
\hline Nicht näher bezeichnete psychische Störungen (F99) & $2 \%$ & $1 \%$ & $1 \%$ & $0 \%$ & $1 \%$ \\
\hline Andere Störung / keine Diagnose gestellt & $6 \%$ & $1 \%$ & $3 \%$ & $5 \%$ & $4 \%$ \\
\hline Total & $100 \%$ & $100 \%$ & $100 \%$ & $100 \%$ & $100 \%$ \\
\hline ( $\mathrm{n}=$ Anzahl antwortende Personen) & $\mathrm{n}=434$ & $\mathrm{n}=372$ & $\mathrm{n}=173$ & $\mathrm{n}=116$ & $\mathrm{n}=1095$ \\
\hline
\end{tabular}

(Quelle: Strukturerhebung, Hochrechnung BASS)

\section{Folgen des Modellwechsels}

Was wären die Folgen einer Ablösung des Delegationsmodells durch ein Anordnungsmodell? Da aktuell noch nicht bekannt ist, wie genau das neue Modell ausgestaltet sein wird, ist es schwierig, eine Prognose zu treffen. Betreffend Kosten kann aber die folgende Aussage gemacht werden: Die Zahlen aus der Studie lassen die Schätzung zu, dass rund 131 Millionen Franken aus dem privaten und öffentlichen Bereich (Selbstzahlung/Zusatzversicherung sowie öffentliche Hand) in den Bereich der Grundversicherung verlagert würden. Dies unter der Voraussetzung, dass sämtliche bisher privat getragenen Kosten neu zulasten der Grundversicherung gehen würden und dass die Gesamtkosten der psychologischen Psychotherapie denjenigen im Jahr 2012 entsprechen.

Das neue Modell, das die vor dem Inkrafttreten des PsyG eingeführte «Übergangslösung» der delegierten Psychotherapie ablösen wird, muss die Arbeitsbedingungen für psychologische Psychotherapeutinnen und -therapeuten verbessern. Nur mit der Ablösung des Delegationsmodells kann sichergestellt werden, dass diese einen nachhaltigen Beitrag zur Verhinderung der Versorgungslücke und damit zur Förderung der psychischen Gesundheit der Schweizer Bevölkerung leisten können. 